\title{
A Conceptual System Architecture for Motivation- enhanced Learning for Students with Dyslexia
}

\author{
Ruijie Wang \\ School of Computer Science and Informatics \\ De Montfort University \\ Leicester, UK \\ P1523357x@my365.dmu.ac.uk
}

Ivar Solheim

Norsk Regnesentral

Oslo, Norway

Ivar.solheim@nr.no

\author{
Liming Chen \\ School of Computer Science and Informatics \\ De Montfort University \\ Leicester, UK \\ liming.chen@dmu.ac.uk
}

\begin{abstract}
Increased user motivation from interaction process leads to improved interaction, resulting in increased motivation again, which forms a positive self-propagating cycle. Therefore, a system will be more effective if the user is more motivated. Especially for students with dyslexia, it is common for them to experience more learning difficulties that affect their learning motivation. That's why we need to employ techniques to enhance user motivation in the interaction process. In this research, we will present a system architecture for motivation-enhanced learning and the detailed process of the construction of our motivation model using ontological approach for students with dyslexia. The proposed framework of the personalised learning system incorporates our motivation model and corresponding personalisation mechanism aiming to improve learning motivation and performance of students with dyslexia. Additionally, we also provide examples of inference rules and a use scenario for illustration of personalisation to be employed in our system.
\end{abstract}

Index Terms - Motivation Model, Personalised Learning, Ontology, Students with Dyslexia

\section{INTRODUCTION}

Students' high level of motivation to learn is connected with their learning success [1][2][3]. User motivation is a user response to the interaction process and is crucial to the success of the interaction process; therefore, recognition of the role of identified motivational factors can play a part in an evaluation of the interface and can be an indicator of how well the design process has addressed user needs and requirements [4][5].

Specifically, learning difficulties such as dyslexia can cause students to reduce their engagement with the education system or drop out [1]. Dyslexia is the most common specific learning difficulties which can affect the way information is learned and processed, occurring neurologically and independently of intelligence [6]. This means they have different ways of thinking and learning requiring specific personalisation of learning service rather than being "cured".

In addition to various learning difficulties such as those regarding reading, writing and speech, dyslexia can also bring with it many psychological effects like lower academic selfesteem or frustration which affect their motivation to learn [7][8]. Most existing user models for dyslexics merely consider dyslexia types and learning difficulties. There is a lack of empirical research investigating motivational determinants and their impact on learning behaviour.

In this research, we examine dyslexic learners' use of assistive learning technology to identify their specific characteristics regarding motivations and barriers faced when using a mobile learning software so that the dyslexic users' motivational needs can be incorporated into user modelling to better adapt assistive learning technology to their specific needs. Our eventual aim is to provide a personalised learning system for students with dyslexia based on our motivation model to improve learning success.

Based on the identified motivational factors from the literature review and our empirical study, a conceptual motivation model has been built [1]. Using the concepts and their relationships in the conceptual model, we will create a more detailed computational user model in which user characteristics obtained from previous studies is structured as a hierarchy of classes with concepts and interdependencies using ontology-based user modelling technique and the formal ontology language.

In the present paper, the related work on personalised learning systems and ontological user modelling will be firstly presented, we will then introduce the design of a personalised system architecture which will incorporate an ontological motivation model and corresponding inference rules to adapt to different individuals' motivational needs. After that, the methodology and process of the motivation modelling will then be described in detail, followed by the mechanism for motivation-based personalisation illustrated by certain key inference rules. An application example of the system will be given along with a use scenario. Finally, the present research will be concluded with the future work being discussed.

\section{RELATED WORK}

Assistive technology can help dyslexic learners to overcome some of the difficulties that they face. There is now a wide range of different technologies available helping with 
many aspects of dyslexia with software and hardware that helps with reading, writing, mathematics and organization. These technologies are becoming increasingly adaptive and more mobile. The research area is this paper is personalised learning systems, which is also called intelligent tutoring systems providing automated adaptive guidance, compared with the other area, educational games as the other approach to technology-enhanced learning according to Peeters [9], as dyslexic adults may find learning games boring which can work well on dyslexic children [10]. Therefore, personalised learning systems have wider target users.

The personalisation process will often require an input of data, a method of algorithmically processing the data and then a final inference or decision that can be used by the system to tailor the experience, configuration, behaviour or output to the user. To customise data and features being delivered to the user or to provide helpful personalisation, the system can perform filtering of the whole data or functionality set. The filtering can be rule-based, content-based, collaborative or a combination of them [11]. Specifically, the rule-based approach requires that pre-defined rules are used to customise data. This however can be inflexible and difficult to update. The content-based approach looks at text data but is not supported for multimedia. The collaborative approach uses interest detection of many people but does not give satisfy results for new users or items. Often a hybrid approach combining all these approaches can give better results but it complicated and difficult to implement [11].

User modelling while beneficial for designing applications are often used for real-time personalisation and adaption of services. User modelling approaches are mainly classified into two categories: knowledge-based approaches and data driven approaches. Data driven user modelling makes use of techniques in machine learning and data mining to examine the relationships among various sensor data and situations surrounding the user, whereas knowledge driven user modelling primarily represents user models via logic rules and applies reasoning engines to eventually infer personalised service from the environment [12]. A commonly adopted knowledge driven approach is the use on ontologies. The ontology-based modelling approach is to produce a structured and reusable model which can be incorporated into a highly adaptable yet reusable system to be easily aligned to the learner's different individual characteristics. It provides a common understanding of the domain [13] and facilitate knowledge sharing and reuse while also very expressive using a web ontology languages. For example, Biletskiy et al [14] has used ontology as a user modelling technique. The IMS Learner Information is presented as many different categories which contribute to the ontology model, including their academic performance, interests and aspirations. Not only is this information fed into the system at the beginning, the learner can also view and edit any of the constituents of the model to correct or update information. The authors emphasised that for good user modelling, it is important for the user to provide accurate and truth information or the model will be rendered useless [14].
The data input for building user models can be from different sources. Razmerita et al has discussed two ways in which user information can be gathered to identify and adapt to the user, either using user supplied data or through system collected data [15]. One example of this in practice is shown in Hatala and Wakkary's Ec(h)o System [16], a museum installation that can adapt to the types of museum visitors based on their interactions with the system. This allows the differentiation between visitors who want detailed information versus busier visitors who just want an overview. A similar method uses hierarchical relationships to depict the user model. Kim and Chan [17] discussed a user interest hierarchy which looks at user attributes but places in a general to specific hierarchy. The user's behaviour on the web site can then be used to populate the hierarchy and algorithm can be used to cluster the different hierarchy themes and levels together.

Existing personalization of learning systems for students with dyslexia helps separate out the needs of different types of dyslexia. Dyslexia is firstly classified by Ingram [18] into three main categories being visuospatial difficulties, speech-sound difficulties and correlating difficulties. The visuospatial difficulties refer to difficulties in distinguishing between letters, syllables and phrase orders, relying on shapes to identify the letters. This may cause issues with identifying the letters and confuse the order of the letters. The speech-sound difficulties refer more to difficulties in spoken language, the forming of sentences and the separation of words into component syllabi. The correlating difficulties are more concerned with writing difficulties where they are not able to link letters to speech sounds [19]. Dyslexia is categorised into five types by Alsobhi et al corresponding to five difficulties experienced by students with dyslexia: reading, writing, speaking, mathematics and memory [19]. Although these types are defined separately, they often occur in combination with an individual possibly having 2 or 3 of these types [19].

Alsobhi et al [20] have attempted to linking dyslexia types and symptoms to the available assistive technologies by summarizing the different existing assistive technologies and how they correlate with the different combinations of the types of dyslexia. The information from the correlation can be used to adapt the assistive technology to the needs of the individual dyslexic depending on the type or combination of types of difficulties that they have. It has also been [19] considered using an ontology approach to address the dyslexia type personalization issue. An ontology web language was used which handles the content and the presentation of the content separately to allow different presentations of the same content to be shown depending on the learning difficulties of the individual. The ontology approach involves splitting up the learning system into classes with dependences on each other, and the classes model the students, their individual dyslexia types and learning styles as well as the learning systems and the hardware and software adaptions that can be employed. This is then used to determine the adaptations and assistive technology that are required. Users can also select preferences that are stored as settings for system features that will benefit their learning experience [19]. 
User modelling and personalisation has been attempted to develop a system that can flexibly adapt to users' learning needs and requirements, and ontological approaches have great potential for this purpose by personalising learning experiences through inferences. However, they lack the ability to provide dyslexic learners with motivation-enhanced learning experiences that takes motivational factors into account, which is an essential aspect of dyslexic learning behaviour. Students' motivation has significant influence on learning performance. For instance, e-learning feedback can improve the level of students' motivation [21][22]. Existing personalised learning systems also lack context-dependent feedback such as providing encouragement and the constant measurement of responses and the corresponding feedback as well compared with having tutors in the classroom; furthermore, most learning systems are developed from a well-intentioned concept without involving wider user groups with diverse strengths and weaknesses [23], there is a lack of suitable mechanisms to ensure that the learning system is designed and personalised most appropriately for the specific needs of dyslexic individuals. Also, the personalization needs to occur in an automated fashion in the big data age to ensure that the data available for each student and for dyslexics as a whole can be used to benefit everyone and provide the best personalization. Therefore, we aim to design a personalised learning system that incorporates learners' motivation models to provide dyslexic students with personalised learning experience to enhance learning motivation and performance.

\section{THE SySTEM ARCHITECTURE}

The motivation-enhanced learning system is designed to be a Moodle add-on or plugin. Fig. 1 basically shows the system architecture and its working principles to perform learning behaviour analysis for modelling of motivation and personalisation of learning materials and techniques. Combining our motivation model and the corresponding inference rules, we will need the system to collect the user data which will fit into with the motivation model to be used for generating inferenced outputs.

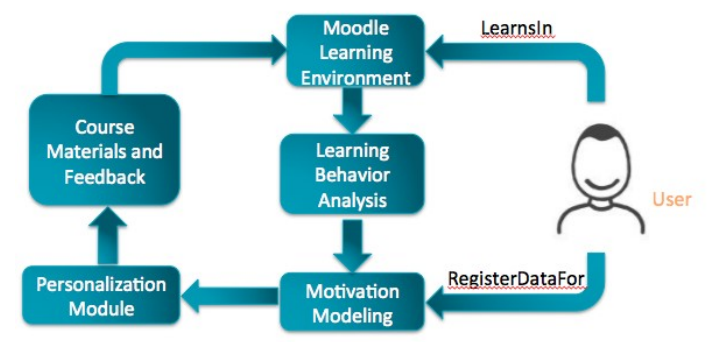

Fig. 1 System architecture for learning monitoring and personalisation.

In the personalisation process, a user model need to be constructed that can then be consumed by the system for personalisation. This user model can potentially be universally used by many different systems. The data can be from a range of different inputs, which can all contribute to the model. Data can either be implicit/dynamic or explicit/static [11]. The static/explicit data is based on user input which can take the form of registration forms, questionnaires or user provided feedback, whereas dynamic/implicit data is derived from the observation of user behaviour, which in computer system can take the form of logs, databases, cookies or information from user choices, selections and browsing habits [11].

In the system, the initial personalisation of learning content will be performed based on a motivation questionnaire and the rules, and then the real-time user behaviour data will be recorded and used to update user's motivation. The user motivation is obtained from questionnaire score and updated through the analysis of user's learning behaviour. According to the real-time motivation, adaptive feedback and course materials will be output to user to sustain and enhance their motivation.

The first extension of the add-on deals with detecting and storing the motivational state of the students. For detecting motivation, we will use the motivation questionnaire, which is a multi-item scale developed based on the conceptual motivation model we constructed before [1]. The motivation questionnaire exceeds the scope of this paper and will not be presented here. We will add the questionnaire to the registration form in Moodle, allowing the calculation of the results of students' motivations and the storage in the motivation model. As indicated by the questionnaire result, we will distinguish the motivational state as strong, moderate and balance, which is similar to the approach to distinguishing the preferences of learning style suggested by literature [21]. In addition to the self-reported data from the questionnaire, another source of data input for detecting motivation is the real-time learning behaviour of the students during their interaction with the system. For example, we will employ eye tracker to record eye movements to be analysed as indicators for user attention and motivation; also, we will record the students learning performance such as quiz score, time spent on the course, etc. to reflect their learning motivation. These real-time data will be analysed and fit into the students' motivation model. The other extension enables the system to automatically provide courses and feedback that adapt to the motivational states of the students. The personalisation module is responsible for accessing the information about students' motivation through the motivation model and calculating the values of each personalisation feature based on motivation, indicating the quantity of the course and the content of feedback that should be provided, similar to the adaptation module adopted by Graf et al [24][25]. Then the suitable course and feedback will be created and presented to the students via the interface in the Moodle learning environment.

Compared to the traditional system, this novel system is user-centric, aiming at providing personalised learning for students with dyslexia. The system takes a user's self-input data to construct a motivation model and records the user's learning state focusing on motivational state to update the model in real time, which is the basis of the filtering to provide personalised service based on individual motivational states to increase the user's intention of using the system to improve learning 
performance. The system can also be adapted to cater for users with other special learning difficulties or needs.

\section{Motivation Modelling PROCESS}

\section{A. Conceptual Modelling}

The approach of conceptual modelling is to initially build the model by integrating the most important motivational factors from existing motivation theories with adjustments to adapt to our research context [1]. Then, an empirical study is conducted with target user to identify the relevant motivational factors in real world scenario and if necessary, leading to the model refinement accordingly.

1) Theoretical Background: Psychological theories can be referred to for establishing an explanatory framework for the users' response [1] and providing insights to help reprioritize design considerations and evaluate how well the system design addresses user's needs and requirements. Human motivation has been studied by psychologists to have multiple factors, and the most important motivation theories and factors most relevant to the research context has been employed to build the present motivation model.

The initial conceptual motivation model is shown in Fig. 2 [1]. The arrows and H1-H9 represents the relations between the model constructs.

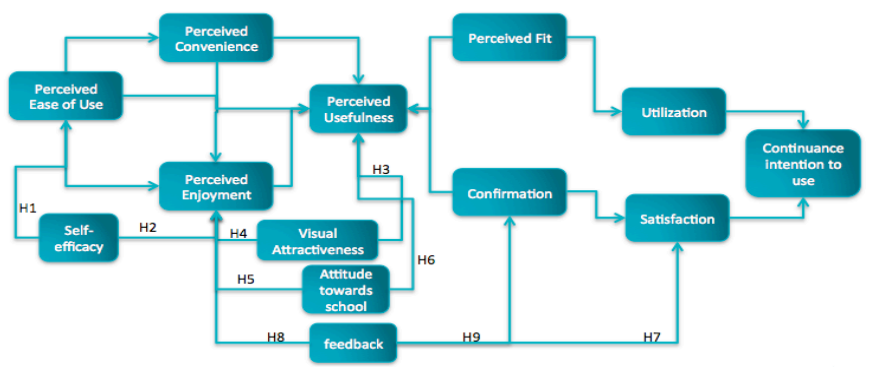

Fig. 2 the initial conceptual motivation model [1]

The first basis of the present motivation model is the extended post acceptance model proposed by Larson et al [1][26], stating that utilization level of an information system is positively related to user's continued intention to use the system and the user's perceived task-technology fit of the system, and the latter is positively connected with perceived usefulness of the system. Similarly, students with dyslexia need to perceive an assistive learning system to fit their learning needs in order to generate use motivation. The second basis of the present model is the extended technology acceptance model proposed by Chang et al [1][27], where perceived convenience and perceived playfulness also play a role in the continuance of use intention of a piece of technology, in addition to perceived usefulness and perceived ease of use. We also noted that students with dyslexia as the target audience in our research are young teenagers with dyslexia, so perceived enjoyment and perceived convenience are also likely to contribute to their intention of use of the tools. Our inspirations also involves technology self-efficacy and computer playfulness, visual attractiveness of the assistive learning system, attitudes towards school and feedback as components with consideration of dyslexic students' specific characteristics [1].

2) Empirical Study: A small-scale empirical study was conducted with a sample of 11 students with dyslexia at the age of 14-16. The study intends to collect all possible motivations and barriers for target user. A clear and holistic view of the context is provided with a prototype of mobile learning software called mYoutime from PLA project[smartlearn] to the participants. Each lesson in mYoutime is less than 15 minutes with text, audio and video. Focusing on a practical application of existing technology allows the users to be observed. The study helps collect first-hand data on the views of dyslexics on learning resources [1]. A between-subject design is adopted with Group A having the version of the learning content that can be read aloud with a button that can be clicked on by users to enable the function and Group B having the other version of the content that cannot be read out [1]. Each participant has 4 learning tasks, then follow-up questions are given on their learning experience and the two versions of feedback to be output to user in mYoutime are compared for all participants. One version immediately shows whether an answer to a quiz is correct or not, but the alternative is prototyped o make the feedback more interesting and probably more encouraging with levels and badges concerning their task performance [1].

Data is analysed with paper coding and a thematic approach, user motivations are identified for participants are then related to corresponding motivation themes retrieved from existing theories. In case no match is found in the prevailing literature, a new theme would be proposed for emerging themes. Secondly, the interviews are coded and analysed using qualitative data analysis software (NVivo 10), to improve the methodological objectivity and reliability [1].

Based on the motivations revealed in the empirical study and on related research into the motivations of using learning systems and dyslexic students' concerns, these factors and interrelationships are proposed and incorporated to refine the initial conceptual motivational model. The refined conceptual motivation model is shown in Fig.3. The emerging motivation themes are shown in green with the new relations N1-N6 in the model being hypothesised according to existing motivation theories, and the themes with dashed lines are those that was not confirmed or examined in the empirical study [1].

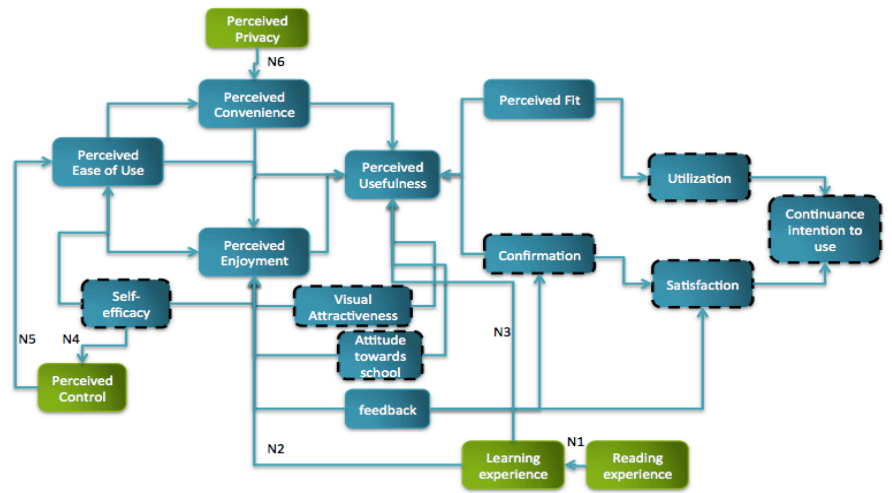

Fig. 3 the refined conceptual motivation model [1]

\section{B. Ontological Modelling:}


Existing ontological user models for personalised learning systems that have been applied to personalised learning systems are mostly restricted to taxonomies of user interests. In particular, there is a distinct lack of incorporating a learner's motivational factors into the user model to support motivationenhanced learning. The present ontology aims to model the motivational needs based on the previously presented conceptual motivation model.

1) Ontology Creation Resources: We adopted an OWLbased design to build computational model. The Protégé5.2 framework is typically employed to edit and update contents. The MotivationState class is built by encoding the built conceptual model with its constructs and interrelationships into ontologies. The overall ontology for students with dyslexia is constructed based on the ontology built by Alsobhi et al [19].

2) An Overview of Ontology: This section presents an overview of the ontology of a learner who has dyslexia in a personalized learning system environment, shown in Fig. 4. We extend the existing ontology by incorporating the motivation model to make the personalisation not only adapt to dyslexic learning style but also base on their motivation state.

The Course Materials and Feedback Class represents the learning contents and other course elements such as quizzes and feedback in-between. The hasObjectives property refers to the objectives of the course.

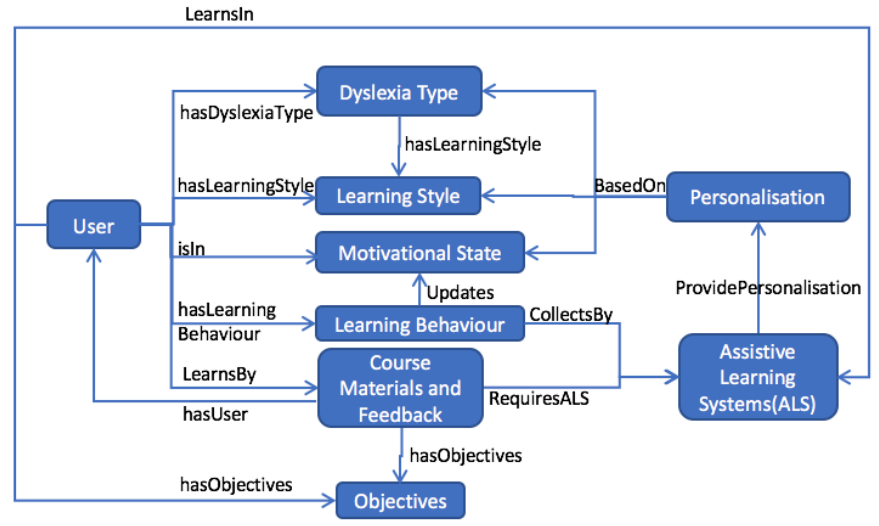

Fig. 4 General framework of the overall ontology with the motivation model

In the ontological motivation model represented by MotivationState class, each student will have a profile with a specific motivational state to be used for personalising the learning service, the student and his or her motivation state is connected by isIn object property. As shown in Fig. 5, an instance of Student class called Student1 has a specific motivational state represented as StudMotivl which is an instance of MotivationState class, and they are connected by isIn Object property. Studmotivl has a value of Level 5 as its data property.

Fig. 6 shows the motivation model produced by a Protégé5.2 plugin called ontoGraf in protégé, where the classes are mainly connected by properties of correlations according to our conceptual motivation model.

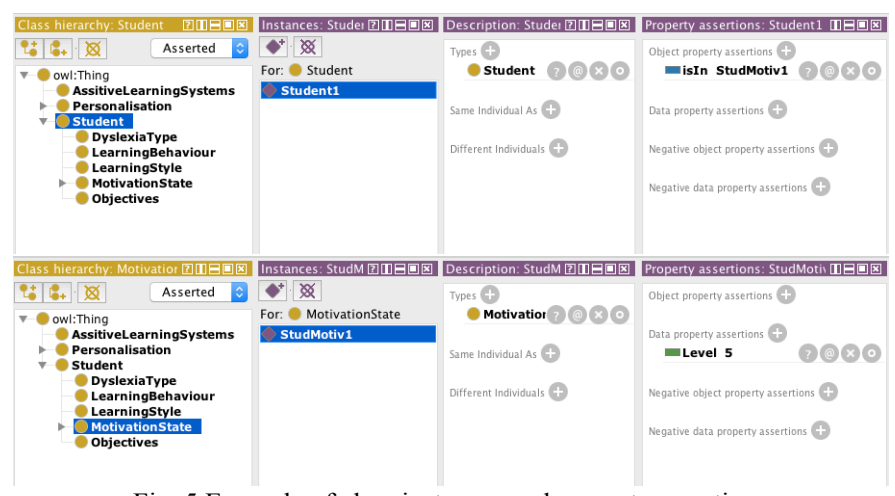

Fig. 5 Example of class instances and property assertions

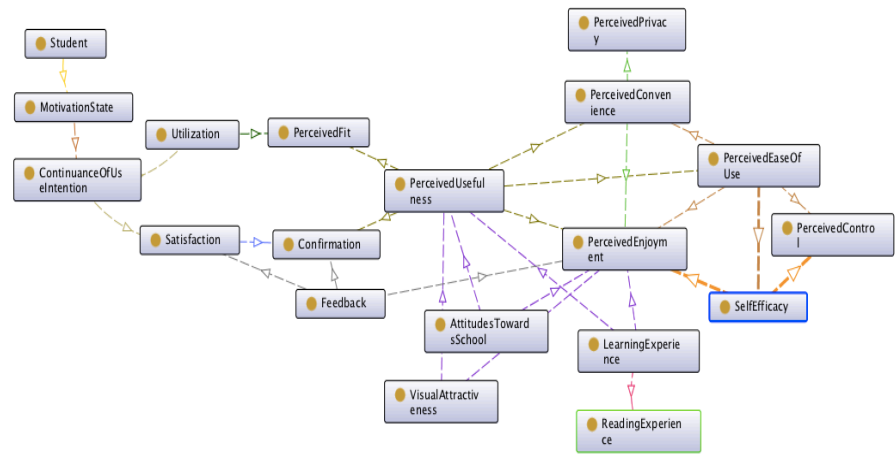

Fig. 6 Ontological motivation model by OntoViz

3) Other Classes and Relationships: The Dyslexia Type and Learning Style classes presented by previous research are adopted in this research [28][29][19], in which Learning Style class is built on Felder and Silverman's Learning Style Model (FSLSM) and each type of dyslexia was matched with the dimensions of FSLSM [19][30]. The Dyslexia Type class reflects the dyslexia difficulties including five properties: reading, writing, speaking, mathematics, memory. The DyslexiaType class are matched with LearningStyle class with the hasLearningStyle property object. Furthermore, the LearningStyle class is composed of the FSLSM components of learning styles-reflective, visual, sensory, sequential, and auditory. The same approach to link dyslexia types to learning styles has been employed by previous research [19].

MotivationState class of the ontology reflects the factors that should be included in the motivation model, incorporating different aspects of motivational needs. Fig. 7 shows the class hierarchy generated by a Protégé5.2 plugin called owlViz.

The Personalisation class (see Fig. 8) is a semantic class to provide a range of personalisation options that allow the system to be adapted to the user's motivational state and other dyslexic needs. The Personalisation class categorises the various solutions and applications that are available into semantic classes." For instance, the first level of the class classifies the solutions into ten generic classes: Reading, Writing, Communication, Hearing, Vision, Mobility, ShortMemory, Organisation, CourseFeedback and CourseQuantity. We adopt the first eight classes proposed by Alsobhi et al [19], which are related to the difficulties of students with dyslexia, while the 
last two classes involve providing personalisation based on user's motivational state. The motivation is suggested to be primarily considered in the quantity of course materials and feedback presented to the student [21][31]. For example, students with higher motivation are more likely to learn faster and learn bigger quantities of the course materials; also, positive feedback can change user's motivational state, as studies have shown the positive correlation between the feedback of the learning progress ("which makes ability perception") and learning engagement [21]. The more the classes in the ontological model is drilled down, the more the system is personalised.

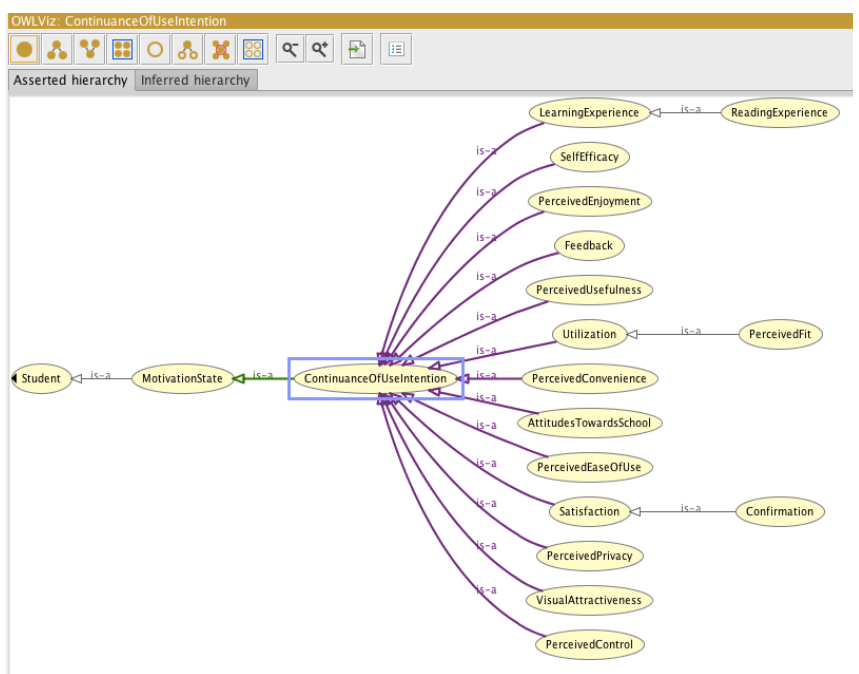

Fig. 7 Hierarchy of the MotivationalState class by owlViz

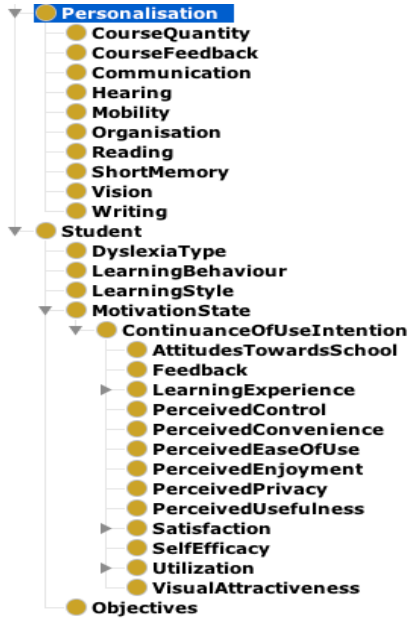

Fig. 8 Class Personalisation and Student with their subclasses in Protégé

\section{THE MECHANISM FOR PERSONALISED LEARNING}

User modelling itself is not sufficient for providing personalised learning, and some mechanism of personalisation is necessary. Once the input data is collected or made available, the data will need to be passed through an algorithm that will provide the conclusions and predictions that will make its way into the model.

The inference rules will be developed in our research and further used for evaluation of our motivation model. The rules will mainly deal with the personalisation mechanism based on motivation model. Therefore, based on the user's data collection including both self-reported data and real-time learning behaviour data combined with the motivation model and inference rules, the system will output personalised learning service. Specifically, in our research, the output will be different quantities of course materials along with feedback to user as discussed in the previous section.

We can employ Semantic density (SD) representing the complexity and semantic quantity of learning objects adopted by previous research [21][32]. For example, each learning object can be assigned to a semantic density between 1 and 5 , the learning objects with $\mathrm{SD}$ value $<=2$ can be presented to students with low motivation, and those with moderate motivation can be assigned to the learning objects with $\mathrm{SD}<=$ 4 totally, and highly motivated students can have learning objects with semantic density value 5 .

We will adopt the principles of feedback intervention indicated by previous research [31], which has proposed the possibility to incorporate a character as a mechanism to deliver varying motivational feedback that could be matched to the perceived motivational state. They also used a character to express motivating spoken feedback concerning the learning domain, adapting its tone by considering the learner model. We can apply emoticons along with the words for providing personalised feedback for their ability of conveying social emotions. Specifically, If the user's motivation is high, the facial expression of the emoticon providing feedback will be happy, else it will be worried. The presented feedback will consider the phenomenon of de-motivation and the learning context suggested by previous research [31]. The emoticon can provide feedback before and after a learning course. Pre-course feedback presents varying facial expression based on the student's motivational state and the student's performance in the previous course. Post-course feedback will be only provided when the student has low motivation during the course. Examples of feedback included: "Believe yourself and take more challenge". In addition, we will also incorporate the factors and strategies to increase extrinsic motivation using badges and levels as rewards of a considered good learning performance.

\section{An ApPlicAtion EXAMPLE}

Jason is a student with dyslexia in a large university who wants to use the learning system. He sets up an initial account in the system by providing username and password, and a learner id is automatically generated and associated with the profile.

Jason is registered as a new user in the system so that he can login the system with his username and password. Once logged in, the learner can view or edit its own profile by submitting a text reader friendly form containing basic 
demographic information and answers to the motivation questionnaire as well as the questions concerning his dyslexic difficulties (in his case, reading) to help the system to adjust to his needs and preferences. All the information he inputs is stored in his learner profile and used to build his own learner model including his motivation model. Once he provides all the information required at registration stage, the system will suggest an initial personalised course materials according to his dyslexia difficulties, learning style and objectives got from his self-input information.

In this paper, we will focus on the illustration on the motivation-based learning personalisation. According to Jason's motivation model, he has a low motivational state. The quantity of course materials is personalised. Each course contains elements including theory, examples, practice, test, index, problem set, case study and group discussion represented with several appropriate learning objects. Learning object educational metadata SD is assigned to each learning object.

His learning content in each learning interface contains one learning object with semantic density $\mathrm{SD}=1$ and it is all the content learner gets in the first step because his level of motivation is low. Thus, this learner will be presented step by step with several learning objects and each time followed with test and appropriate feedback.

During the process of his learning, eye tracker records his eye movements and calculates the percentage of time he spent on looking at different areas. This is taken into account together with his learning performance when it comes to providing him with appropriate feedback and suggests the amount of learning materials right after he finishes the current stage of learning materials.

As Jason's motivation is low, he needs permanent positive feedback and encouragement [21]. Emoticon along with the words are employed before and after a learning activity. Before a learning course, a positive emoticon with the words "Have a try and you will make progress!" will be given to Jason. He finishes the learning activity and gets a high score in the selfassessment quiz, but the eye tracking data shows he still has spent a non-ignorable amount of time looking out of the area of learning content. After the learning course, he gets the informative feedback about the scores and badges obtained from the learning activity, ad a positive emoticon with the words "Well done! believe yourself and take more challenge" is presented to him.

He can also update his profile anytime he logs into the system. When he updates the model, the model is saved in database and also in learner model RDF file. The quantity of course materials and feedback will be personalised further to adapt to his latest motivation model.

\section{CONCLUSION AND FUTURE WORK}

In this research, we proposed an ontological motivation model for students with dyslexia with demonstration of the construction process. We also displayed our design of the learning system along with the personalisation illustration for further application and evaluation.
A heuristic evaluation will be performed by educational experts and ontological experts. The process of evaluation and the outcome will be described in a later paper.

We will apply data-driven approach to the modelling. Firstly, we will employ a multi-item questionnaire developed based on our motivation model; in addition, we will conduct experiment to collect data on students' learning behaviour and performance to train the connections between the constructs in our model.

Furthermore, we will refine and complete the pre-defined rules to be implemented along with our ontological model in the system. Following that, we will perform evaluation study to test our model and personalisation mechanism.

\section{REFERENCES}

[1] R. Wang, L. Chen, I. Solheim, T. Schulz, and A. Ayesh, "Conceptual Motivation Modeling for Students with Dyslexia for Enhanced Assistive Learning," in SmartLearn '17 Proceedings of the 2017 ACM Workshop on Intelligent Interfaces for Ubiquitous and Smart Learning, 2017, pp. 11-18.

[2] W. E. Blank, S. H. Harwell, U. S. D. of Education. Office of Vocational, and A. Education, Promising Practices for Connecting High School to the Real World. University of South Florida, 1997.

[3] P. C. Dev, "Intrinsic motivation and academic achievement: What does their relationship imply for the classroom teacher?," Remedial Spec. Educ., vol. 18, no. 1, pp. 12-19, 1997.

[4] W. Shane and P. David, "How Is It for You? (A Case for Recognising User Motivation in the Design Process)," in Affect and Emotion in Human-Computer Interaction: From Theory to Applications, P. Christian and B. Russell, Eds. Springer Berlin Heidelberg, 2008, pp. 130--141.

[5] C. Peter and R. Beale, Eds., Affect and Emotion in HumanComputer Interaction: From Theory to Applications. Springer Science \& Business Media, 2008.

[6] J. Backhouse, Essential Health and Safety Study Skills. Routledge, 2013.

[7] N. Firth, E. Frydenberg, C. Steeg, and L. Bond, "Coping successfully with dyslexia: An initial study of an inclusive schoolbased resilience programme," Dyslexia, vol. 19, no. 2, pp. 113-130, 2013.

[8] R. Burden, "Is Dyslexia Necessarily Associated with Negative Feelings of Self-worth? A Review and Implications for Future Research," Dyslexia, vol. 14, no. 3, pp. 188-196, 2008.

[9] M. Peeters, Personalized Educational Games - Developing agentsupported scenario-based training. Ridderprint, Ridderkerk, 2014.

[10] Y. Griffiths and M. Stuart, "Reviewing evidence-based practice for pupils with dyslexia and literacy difficulties," J. Res. Read., vol. 36, no. 1, pp. 96-116, 2013.

[11] Z. Jrad, M.-A. Aufaure, and M. Hadjouni, "A Contextual user model for Web personalization," Web Inf. Syst. Eng. 2007 Work., pp. 350-361, 2007.

[12] A. Briassouli, J. Benois-Pineau, and A. Hauptmann, Eds., Health Monitoring and Personalized Feedback using Multimedia Data. Springer, 2015.

[13] A. Andrejko, M. Barla, and M. Bieliková, “Ontology-based User Modeling for Web-based Information Systems," in Advances in Information Systems Development, 2007, pp. 457-468.

[14] Y. Biletskiy, H. Baghi, I. Keleberda, and M. Fleming, "An adjustable personalization of search and delivery of learning objects to learners," Expert Syst. Appl., vol. 36, no. 5, pp. 9113-9120, 2009.

[15] L. Razmerita, A. Angehrn, and A. Maedche, "Ontology-based User Modeling for Knowledge Management Systems," in Proceedings of the User Modeling Conference, 2003, vol. 2702, pp. 213-217.

[16] R. Wakkary, M. Hatala, and K. Newby, "ec ( h ) o : Ecologies For Designing Playful Interaction," pp. 1-6, 2006.

[17] H. R. Kim and P. K. Chan, "Learning implicit user interest 
hierarchy for context in personalization," Appl. Intell., vol. 28, no. 2, pp. $153-166,2008$

[18] T. Ingram, The dyslexic child. The Practitioner 192, 1964

[19] A. Y. Alsobhi, N. Khan, and H. Rahanu, "Personalised learning materials based on dyslexia types: Ontological approach," Procedia Comput. Sci., vol. 60, no. 1, pp. 113-121, 2015.

[20] A. Y. Alsobhi, N. Khan, and H. Rahanu, "Toward linking dyslexia types and symptoms to the available assistive technologies," Proc. IEEE 14th Int. Conf. Adv. Learn. Technol. ICALT 2014, pp. 597 598, 2014.

[21] D. Milosevic, M. Brkovic, and D. Bjekic, "Designing lesson content in adaptive learning environments," Technol. Learn. (, pp. 1-9, 2006.

[22] D. J. Mac Iver, D. J. Stipek, and D. H. Daniels, "Explaining withinsemester changes in student effort in junior high school and senior high school courses.," J. Educ. Psychol., vol. 83, no. 2, pp. 201$211,1991$.

[23] I. Smythe, Dyslexia in Digital Age: Making IT Work (1). Continuum, 2010.

[24] S. Graf and K. Kinshuk, "Providing adaptive courses in learning management systems with respect to learning styles," Proc. E-Learn World Conf. E-Learning Corp. Gov. Heal. High. Educ. 2007, vol. 17, no. 1, pp. 2576-2583, 2007.

[25] S. Graf, Kinshuk, and C. Ives, "A Flexible Mechanism for Providing Adaptivity Based on Learning Styles in Learning Management Systems," Adv. Learn. Technol. (ICALT), 2010 IEEE 10th Int. Conf., pp. 30-34, 2010.

[26] T. J. Larsen, A. M. Sørebø, and Ø. Sørebø, "The role of tasktechnology fit as users' motivation to continue information system use," Comput. Human Behav., vol. 25, no. 3, pp. 778-784, 2009.

[27] C. C. Chang, C. Liang, C. F. Yan, and J. S. Tseng, "The Impact of College Students' Intrinsic and Extrinsic Motivation on Continuance Intention to Use English Mobile Learning Systems," Asia-Pacific Educ. Res., vol. 22, no. 2, pp. 181-192, 2013.

[28] N. Capuano, M. Gaeta, A. Micarelli, and R. Tre, "Automatic Student Personalization in Preferred Learning Categories," no. July, 2005.

[29] H. Shi, O. Rodriguez, Y. Shang, and S. Chen, "Integrating adaptive and intelligent techniques into a webbased environment for active learning," in Intelligent Systems: Technology and Applications, CRC Press, 2003, pp. 229-260.

[30] R. M. Felder and L. K. Silverman, "Learning and teaching styles in engineering education," J. Eng. Educ., vol. 78, no. 7, pp. 674-681, 1988

[31] G. Rebolledo-Mendez, R. Luckin, and B. Boulay, "Designing adaptive motivational scaffolding for a tutoring system," New Perspect. Affect Learn. Technol. SE - 12, vol. 3, pp. 155-168, 2011.

[32] “Advanced Distributed Learning: SCORM," 2004. [Online]. Available: http://www.adlnet.org/index.cfm. 